\title{
Impact of supplementation with a food-derived microbial community on obesity-associated inflammation and gut microbiota composition
}

\author{
Marianna Roselli, Chiara Devirgiliis* (1), Paola Zinno, Barbara Guantario, Alberto Finamore, Rita Rami
} and Giuditta Perozzi

\begin{abstract}
Background: Obesity is a complex pathology associated with dysbiosis, metabolic alterations, and low-grade chronic inflammation promoted by immune cells, infiltrating and populating the adipose tissue. Probiotic supplementation was suggested to be capable of counteracting obesity-associated immune and microbial alterations, based on its proven immunomodulatory activity and positive effect on gut microbial balance. Traditional fermented foods represent a natural source of live microbes, including environmental strains with probiotic features, which could transiently colonise the gut. The aim of our work was to evaluate the impact of supplementation with a complex foodborne bacterial consortium on obesity-associated inflammation and gut microbiota composition in a mouse model.
\end{abstract}

Methods: C57BL/6J mice fed a 45\% high fat diet (HFD) for 90 days were supplemented with a mixture of foodborne lactic acid bacteria derived from the traditional fermented dairy product "Mozzarella di Bufala Campana" (MBC) or with the commercial probiotic GG strain of Lactobacillus rhamnosus (LGG). Inflammation was assessed in epididymal white adipose tissue (WAT) following HFD. Faecal microbiota composition was studied by next-generation sequencing.

Results: Significant reduction of epididymal WAT weight was observed in MBC-treated, as compared to LGG and control, animals. Serum metabolic profiling showed correspondingly reduced levels of triglycerides and higher levels of $\mathrm{HDL}$ cholesterol, as well as a trend toward reduction of LDL-cholesterol levels. Analysis of the principal leucocyte subpopulations in epididymal WAT revealed increased regulatory T cells and CD4 ${ }^{+}$cells in MBC microbiotasupplemented mice, as well as decreased macrophage and CD8 ${ }^{+}$cell numbers, suggesting anti-inflammatory effects. These results were associated with lower levels of pro-inflammatory cytokines and chemokines in WAT explants. Faecal bacterial profiling demonstrated increased Firmicutes/Bacteroidetes ratio in all mice groups following HFD.

Conclusions: Taken together, these results indicate a protective effect of MBC microbiota supplementation toward HFD-induced fat accumulation and triglyceride and cholesterol levels, as well as inflammation, suggesting a stronger effect of a mixed microbial consortium vs single-strain probiotic supplementation. The immunomodulatory activity exerted by the MBC microbiota could be due to synergistic interactions within the microbial consortium, highlighting the important role of dietary microbes with yet uncharacterised probiotic effect.

Keywords: Chronic inflammation, Fermented dairy, Foodborne microbiota, White adipose tissue, High fat diet

* Correspondence: chiara.devirgiliis@crea.gov.it

Food and Nutrition Research Centre, Council for Agricultural Research and

Economics (CREA), Via Ardeatina 546, 00178 Rome, Italy

(c) The Author(s). 2017 Open Access This article is distributed under the terms of the Creative Commons Attribution 4.0 International License (http://creativecommons.org/licenses/by/4.0/), which permits unrestricted use, distribution, and reproduction in any medium, provided you give appropriate credit to the original author(s) and the source, provide a link to the Creative Commons license, and indicate if changes were made. The Creative Commons Public Domain Dedication waiver (http://creativecommons.org/publicdomain/zero/1.0/) applies to the data made available in this article, unless otherwise stated. 


\section{Background}

Obesity is a chronic, multifactorial disorder reaching epidemic proportions globally, affecting persons of virtually all ages in both developed and developing countries $[1,2]$. Promoted by a combination of genetic predisposition, nutritional excess, and sedentary lifestyle, obesity is primarily characterised by increased fat mass, accompanied by development of related disorders [3-5]. Expansion of the adipose organ, mainly affecting white adipose tissue (WAT), results in adipocyte dysfunction. WAT has been increasingly considered not only a metabolic organ, but also an active endocrine tissue, as it secretes a large number of peptide hormones called adipokines, such as leptin and adiponectin, that operate in a complex network and actively communicate with other organs $[6,7]$. Secretion by the adipose organ is disturbed in obesity, as adipokine release is dysregulated and associated with production of several inflammation mediators. For this reason, the adipose tissue is considered to be a major contributor to obesity-linked low-grade chronic inflammation [8]. The inflammatory process involves impairment of both the innate and adaptive immune system and is triggered by local secretion of inflammatory cytokines and chemokines such as tumour necrosis factor- $\alpha$ (TNF- $\alpha$ ), interleukin-6 (IL-6), monocyte chemoattractant protein (MCP)-1, and Regulated on Activation Normal T cell Expressed and Secreted (RANTES). These mediators recruit immune cells from blood vessels, such as lymphocytes and macrophages, which in turn massively infiltrate the adipose tissue [9]. Indeed, high levels of inflammatory cells such as $\mathrm{T} \mathrm{CD}^{+}$lymphocytes and activated M1 macrophages are found in obese WAT, accompanied by decreased levels of $\mathrm{CD} 4{ }^{+} \mathrm{CD} 25^{+}$Foxp $3^{+}$ regulatory $\mathrm{T}$ (Treg) cells, a key population in maintaining immunological tolerance and immune homeostasis [10-12]. This inflammatory status, arising locally and then becoming systemic, triggers the onset of other diseases frequently associated with obesity such as the metabolic syndrome, characterised by visceral obesity, high blood pressure, insulin resistance, high circulating triglyceride levels, and low HDL cholesterol, leading in turn to increased risk of cardiovascular diseases [13-16].

The gut microbiota has recently attracted much attention as a crucial factor associated with obesity [17]. Alterations of intestinal microbial composition, in terms of bacterial phyla and classes associated with improved energy extraction from the undigested dietary carbohydrate component, were identified in obese human subjects and animal obesity models, with consequent impact on host metabolism and energy storage [18]. Both diet- and genetically induced obesity were shown to associate with imbalance in the relative proportion of Gram-negative Bacteroidetes and Gram-positive Firmicutes, the two major phyla of gut bacteria, with the latter prevailing in obese subjects [19]. However, imbalance in these two bacterial phyla is not sufficient by itself to determine the obesity phenotype. Other factors, such as diet, pre- and probiotic supplementation, antibiotics, surgery, and faecal transplantation, can impact the overall metabolic capacity of the gut microbiome [20]. Within this context, dietary interventions aimed at promoting selection of beneficial intestinal microbes could represent a powerful strategy to counteract obesityassociated intestinal dysbiosis. There is growing evidence that probiotic and/or prebiotic supplementation can positively modulate gut microbiota, thus representing important assets in the management of obesity [21]. The probiotic component of the gut microbiota can confer health benefits to the host mainly acting on immunomodulation and positively influencing intestinal microbial balance [22]. Probiotic supplementation was therefore suggested to be able to counteract obesity-associated immune alterations and microbial imbalance [23-25]. As an alternative to commercially available probiotic strains, a natural source of live bacteria is represented by fermented foods, which also confer the advantage of providing the host with a complex microbiota containing several environmental strains with potential probiotic features, such as the capability to transiently colonise animal and human gut and interact with the resident gut microbiota, mainly at a trophic level [26]. Increasing scientific interest in fermented foods was also recently boosted by their possible use as models for more complex microbiota such as the gut [27]. The most relevant foodborne lactic acid bacteria (LAB) belong to the Lactobacillus, Lactococcus, Streptococcus, Pediococcus, and Leuconostoc genera. Several LAB species are also highly represented within the resident gut microbiota of healthy humans. Lactobacillus species, in particular, are abundant both in food and in the gut [28].

The aim of our work was to evaluate the impact of supplementation with a complex foodborne bacterial community on obesity-associated inflammation, as well as on gut microbiota composition. For this purpose, we used a mouse model of high fat diet (HFD)-induced obesity, comparing the effect of supplementation with a mixture of natural LAB strains derived from the traditional fermented dairy product "Mozzarella di Bufala Campana" (MBC) [29] and with the well-characterised probiotic GG strain of Lactobacillus rhamnosus (LGG). The MBC bacterial consortium was dominated by Lactobacillus delbrueckii, Lactobacillus fermentum, and Leuconostoc lactis [30]. LGG was used as probiotic control on the basis of its proven beneficial effects in the prevention of obesity [31, 32]. 


\section{Methods}

\section{Experimental design, animals, and diets}

Six-week-old C57BL/6J male mice, obtained from Charles River Laboratories (Como, Italy), were kept at $23{ }^{\circ} \mathrm{C}$ with a 12-h light-dark cycle and fed ad libitum with a standard laboratory diet (4RF21, Mucedola, Milano, Italy, www.mucedola.it). Mice had free access to food and water throughout the experiments. Food intake and body weight were recorded every other day. After 1 week of adaptation, animals were randomly divided into three groups (five mice per group) and orally supplemented for 15 days with $1 \times 10^{9} \mathrm{CFU} /$ day of a mixture of natural LAB strains extracted from MBC [29] or with the probiotic strain LGG. Phosphate-buffered saline (PBS) supplementation was used as control (CTRL). After 15 days, all mice were shifted to HFD (http:// www.envigo.com/resources/data-sheets/06415.pdf, 44.8\% total calories from fat, designed with similarities to Research Diets, Inc., formula D12451 and provided by Mucedola) while continuing to receive bacterial supplementation for 90 additional days. Due to logistic reasons related to the number of animals that could be handled at the same time, the experimental design envisioned two rounds of treatment, 2 weeks apart from each other, in which the two groups of mice, of the same age, were fed the same batches of diets. Therefore, the second group of mice was not aimed at testing reproducibility, but rather at increasing the number of treated animals. Statistical analysis of the results included all animals subjected to the same supplementation protocol, irrespective of their treatment within experimental period 1 or 2. At the end of the experimental period, mice were anaesthetised by intraperitoneal injection of pentobarbital $(10 \mathrm{mg} / \mathrm{kg})$ following overnight fasting, blood was drawn via cardiac puncture, and epididymal WAT was excised, weighed, and immediately placed in ice-cold PBS under sterile conditions. Serum was prepared from blood and stored at $-80{ }^{\circ} \mathrm{C}$ until further analysis. Faeces were collected and stored at $-80{ }^{\circ} \mathrm{C}$ for microbiological analysis at the following time points: to (beginning of bacterial treatments), $\mathrm{t} 15$ (shift to HFD) and $\mathrm{t} 105$ (90 days on HFD). The experimental protocol and sampling times are summarised in Fig. 1.

\section{Bacterial preparations}

MBC is a traditional Italian fermented cheese with PDO designation (Product of Designated Origin, EEC Regulation no. 1107). It is consumed fresh, within 2 weeks from production, and it contains high titres of live bacteria [29]. To prepare the MBC microbiota, $10 \mathrm{~g}$ of cheese samples were diluted in $90 \mathrm{ml}$ sodium citrate solution $(2 \% w / v)$ and homogenised in a BagMixer400 (Interscience, France), as previously described [30]. To standardise the bacterial inoculum to be administered to

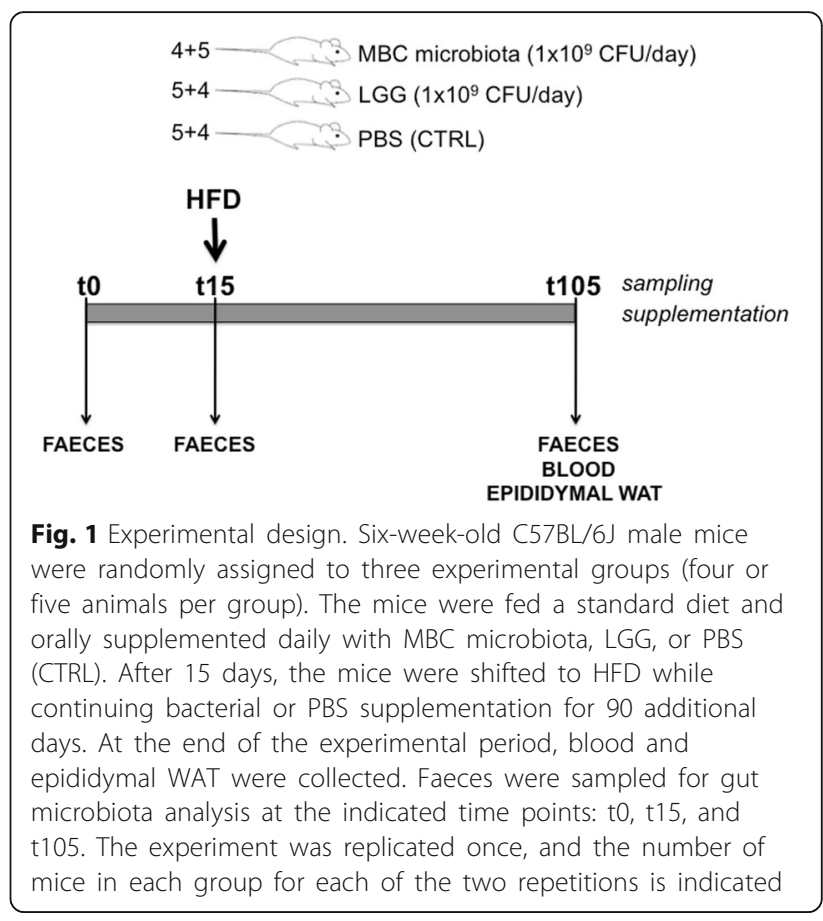

mice, the MBC homogenate was entirely used as a single inoculum in 21 of De Man Rogosa Sharpe (MRS) medium (Oxoid Ltd., England) and incubated at $37{ }^{\circ} \mathrm{C}$ for $48 \mathrm{~h}$ under anaerobic conditions (Anaerocult A, Merck, Germany) to obtain a final bacterial titre of about $1.5 \times 10^{9} \mathrm{CFU} / \mathrm{ml}$. The resulting bacterial suspension was divided in aliquots containing $1 \times 10^{9} \mathrm{CFU}$ each, stored at $-80{ }^{\circ} \mathrm{C}$ in $20 \%(v / v)$ glycerol, and thawed daily for oral administration to mice, following washing, resuspension in $1 \times \mathrm{PBS}$, and mixing with small amounts of minced feed.

The LGG strain ATCC53103 was grown, prepared, and orally given to mice as described above for $\mathrm{MBC}$ microbiota.

\section{Serum metabolic measurements}

The following plasma parameters were analysed: glucose (Glucose Liquid kit, Sentinel Diagnostics, Milan, Italy), HDL and LDL cholesterol (Max Discovery HDL and LDL Cholesterol Assay Kits, Bioo Scientific, Austin, TX), and triglycerides (Triglycerides Liquid kit, Sentinel Diagnostics). The adiponectin was quantified by ELISA (Biorbyt, Cambridge, UK). Analyses were conducted on a subset of five samples for each treatment, due to technical issues related to serum withdrawal or hemolysis.

\section{Immune cell isolation and staining}

Macrophages and lymphocytes were isolated from the epididymal WAT stromal vascular fraction (SVF), according to [33], as several populations of immune cells are well known to reside in the SVF. The following 
monoclonal antibodies, purchased from eBioscience (San Diego, CA), were used in this study: FITC anti-CD3 (clone 500A2), PE anti-CD8 (clone 53-6.7), PE-Cy5 antiCD4 (clone RM4-5), FITC anti-CD11b (clone M1/70), $\mathrm{PE}$ anti-F4/80 (clone BM8), PerCP-Cy5.5 anti-CD45 (clone 30-F11), and anti-CD16/CD32 (clone 93). Briefly, $1 \times 10^{6}$ cells, resuspended in FACS labelling buffer (PBS with $2 \mathrm{mM}$ EDTA and 1\% foetal calf serum), were preincubated for 20 min with anti-CD16/CD32 to avoid non-specific binding, then washed and labelled with the appropriate mixture of antibodies for $30 \mathrm{~min}$, centrifuged, and resuspended in FACS labelling buffer. Flow cytometry analysis was performed using a FACSCalibur flow cytometer (BD Biosciences, Milan, Italy). To exclude dead/dying cells that could non-specifically bind antibodies, leukocytes were gated according to forward and side scatter. The percentage of $\mathrm{T}$ helper and cytotoxic cells was calculated on lymphocyte gate $\left(\mathrm{CD}^{+}\right)$, whereas the $\mathrm{CD}_{11 \mathrm{~b}^{+}}$and $\mathrm{F} 4 / 80^{+}$cell subsets were calculated on the leukocyte gate $\left(\mathrm{CD} 45^{+}\right)$. Treg cell $(\mathrm{CD} 4$ ${ }^{+} \mathrm{CD} 25^{+} \mathrm{Foxp}^{+}$) analysis was performed with a specific kit (eBioscience, San Diego, CA) staining CD4 (FITC), CD25 (PE) and transcription factor Foxp3 (PE-Cy5), according to the manufacturer's instructions. The percentage of $\mathrm{CD} 25^{+} \mathrm{Foxp}^{+}{ }^{+}$cells was calculated on lymphocyte $\mathrm{CD}^{+}$gate. For all analyses, at least 10.000 events were acquired and analysed using the CellQuest software (BD Biosciences, Milan, Italy).

\section{Cytokine and chemokine secretion in WAT explants}

WAT explant cultures were established essentially as described by [34]. Briefly, epididymal WAT was dissected, weighed, minced, and placed into 12-well tissue culture plates (Corning, Milan, Italy) at $120 \mathrm{mg} /$ well, with either $1 \mathrm{ml}$ T cell activation medium (complete DMEM containing $3.7 \mathrm{~g} / \mathrm{l} \mathrm{NaHCO}, 10 \%$ heat-inactivated foetal calf serum, $4 \mathrm{mM}$ glutamine, $1 \%$ non-essential amino acids, $10^{5} \mathrm{U} / \mathrm{l}$ penicillin and $100 \mathrm{mg} / \mathrm{l}$ streptomycin, $5 \mathrm{ng} / \mathrm{ml}$ phorbol 12-myristate 13-acetate (PMA), and $1 \mathrm{ng} / \mathrm{ml}$ ionomycin) or control medium (complete DMEM without ionomycin and PMA). All reagents were from Euroclone (Milan, Italy), except for ionomycin and PMA, which were from Sigma (Milan, Italy). Conditioned media were collected after $24 \mathrm{~h}$ of culture at $37{ }^{\circ} \mathrm{C}$ in an atmosphere of $5 \% \mathrm{CO}_{2} / 95 \%$ air at $90 \%$ relative humidity and stored at $-80{ }^{\circ} \mathrm{C}$ until further analysis. The levels of cytokines and chemokines were analysed using Bio-plex/Luminex technology (mouse magnetic Luminex screening assay, Labospace, Milan) or ELISA assays (Affymetrix, eBioscience, San Diego, CA). The following cytokines and chemokines were simultaneously detected by Luminex technology in $50 \mu$ undiluted samples: interferon gamma-induced protein (IP)-10, granulocyte macrophage-colony stimulating factor (GM-CSF),
Regulated on Activation-Normal T cell Expressed and Secreted (RANTES), interleukin (IL)-23, IL-4, and IL-10. The following cytokines were analysed by ELISA $(100 \mu \mathrm{l}$ samples): tumour necrosis factor (TNF)- $\alpha$, interferon (IFN)- $\gamma$, IL-17A, and IL-6. For these latter two cytokines, samples were diluted 1:500, as the readings by Luminex assays for IL-17A and IL-6 were out of range.

\section{DNA extraction from faecal samples}

Total DNA was extracted from $80 \mathrm{mg}$ faecal samples with QIAamp DNA Stool Mini Kit (Qiagen, Hilden, Germany) according to manufacturer's instructions. Qiagen DNA extraction method used in this work was chosen as it was listed among the most reproducible kits, ensuring minimal influence on next-generation sequencing (NGS) data analysis [35].

\section{NGS analysis}

NGS was performed on faecal DNA samples from four animals for each of the three experimental groups, at the three time points indicated in Fig. 1, namely t0, t15, and t105 (total number of samples $=36$ ). Partial 16S rRNA gene sequences were amplified using primer pair Probio_Uni and /Probio_Rev, which targets the V3 region of the gene and sequenced at the DNA sequencing facility of GenProbio srl (www.genprobio.com) using a MiSeq (Illumina). Primers and protocols, including amplicon checks, were as described in [36]. Individual sequence reads were filtered with the Illumina software to remove low quality and polyclonal sequences. All Illumina quality-approved, trimmed, and filtered data were exported as .fastq files and processed using a custom script based on the QIIME software suite [37]. Quality control retained sequences $140-400$ bp long, with mean sequence quality score $>20$, and truncation at first base if a low quality rolling 10-bp window was found. Presence of homopolymers $>7$ bp and sequences with mismatched primers were omitted. To calculate downstream diversity (alpha and beta diversity indices, UniFrac analysis), 16S rRNA operational taxonomic units (OTUs) were defined at $\geq 97 \%$ sequence homology using uclust [38]. All reads were classified to the lowest possible taxonomic rank using QIIME and a reference dataset from the SILVA database [39]. Similarities between samples were calculated by unweighted UniFrac [40]. The range of similarities is calculated between the values 0 and 1 . Principal Coordinate Analysis (PCoA) was applied using the UniFrac program.

\section{Statistical univariate analysis}

Values in graphs and tables represent means \pm SD. Prior to analysis, normal distribution and homogeneity of variance of all variables were assumed with Shapiro-Wilk's and Levene's tests, respectively. Statistical significance 
was evaluated by one-way ANOVA or by ANCOVA, followed by post hoc Tukey honestly significant difference (HSD) test. Differences with $P$ values $<0.05$ were considered significant. Statistical univariate analysis was performed with the "Statistica" software package (version 5.0; Stat Soft Inc., Tulsa, OK).

\section{Statistical multivariate analysis}

Non-supervised principal component analysis (PCA) of WAT immunological profiles (leukocyte subpopulations and cytokine/chemokine secretion) was performed with Past software, version 2.17c [41]. Data were collected in a matrix of 27 rows (number of animals) and 15 columns (number of variables) and were auto-scaled by mean-centring and normalised by standard deviation. Pearson's correlation coefficients between variables and principal components, as well as statistical significance of the correlation, were also calculated.

\section{Results}

Bacterial supplementation affects epididymal WAT weight and metabolic parameters

Body and WAT weight values in the three groups of mice are shown in Table 1 in comparison with food and energy intakes. As expected, HFD feeding induced significant weight increase in all groups, leading to comparable body weight and weight gain values by the end of the experimental period. Nevertheless, significant reduction of WAT weight $(P<0.05)$ was observed in MBC-treated animals, as compared to LGG and CTRL mice. Food and energy intake were similar in the three mice groups. To account for a possible influence of food intake on WAT weight, ANCOVA analysis was performed, considering WAT weight as the dependent variable, treatment as the independent variable, and food intake as the covariate. The results confirmed that WAT weight reduction in the $\mathrm{MBC}$ group as compared to LGG and CTRL could not be attributable to differential food intake. Supplementing with the foodborne MBC microbiota also led to reduced serum levels of

Table 1 Body weight, epididymal WAT weight, and food and energy intake from HFD of MBC, LGG, or CTRL mice

\begin{tabular}{llll}
\hline & MBC & LGG & CTRL \\
\hline Body weight (g) & & & \\
$\quad$ Initial & $19.19 \pm 1.44$ & $19.41 \pm 1.79$ & $18.80 \pm 0.89$ \\
$\quad$ Final & $31.75 \pm 1.97$ & $31.88 \pm 2.13$ & $30.63 \pm 3.35$ \\
$\quad$ Gain & $11.61 \pm 1.94$ & $12.47 \pm 2.55$ & $12.36 \pm 2.63$ \\
WAT weight (g) & $1.35^{* \#} \pm 0.31$ & $1.78 \pm 0.30$ & $1.89 \pm 0.49$ \\
Food intake (g/day) & $2.40 \pm 0.66$ & $2.54 \pm 0.62$ & $2.48 \pm 0.63$ \\
Energy intake from & $10.80 \pm 0.60$ & $11.39 \pm 0.69$ & $11.26 \pm 1.05$ \\
HFD (Kcal/day) & & & \\
\hline${ }^{*} P<0.05$ versus CTRL (ANOVA); ${ }^{\#} P<0.01$ versus CTRL and LGG (ANCOVA)
\end{tabular}

triglycerides, coupled with higher levels of HDL cholesterol $(P<0.05$ and $P<0.001$, respectively), and a trend toward decreased LDL cholesterol $(P=0.05)$ as compared to the CTRL group (Table 2). Serum metabolic parameters of LGG-treated mice displayed a similar but milder effect, with a trend toward reduced triglyceride levels $(P=0.05)$ and increased HDL-cholesterol levels $(P<0.05)$. No significant differences were detected among the three groups of mice concerning fasting glucose and adiponectin levels.

\section{WAT immunological profiles highlight the anti-} inflammatory effect of MBC microbiota supplementation Flow cytometry analysis of the main leukocyte subpopulations in epididymal WAT (Fig. 2) revealed increased numbers of the immune homeostasis regulator $\mathrm{CD} 4^{+}$ CD25 ${ }^{+}$Foxp $^{+}$Treg cells (Fig. 2a, $P<0.001$ vs CTRL and $P<0.01$ vs LGG) and $\mathrm{CD}^{+}{ }^{+} \mathrm{T}$ lymphocytes (Fig. 2b, $P<0.001$ vs CTRL) in MBC microbiota-supplemented mice, accompanied by decreased pro-inflammatory $\mathrm{CD}^{+}$ T lymphocytes (Fig. 2b, $P<0.001$ vs CTRL), CD11b ${ }^{+}$activated leukocytes and $\mathrm{F} 4 / 80^{+}$macrophages (Fig. 2c, $P<0.001$ and $P<0.01$ vs CTRL, respectively), suggesting that $\mathrm{MBC}$ supplementation associates with an overall antiinflammatory effect. LGG treatment also positively affected WAT leukocyte subpopulations in terms of increased percentage of Treg $\left(P<0.05\right.$ vs CTRL) and $\mathrm{CD} 4^{+}$cells $(P<0.001$ vs CTRL $)$ and decreased $\mathrm{CD}^{+}$cells $(P<0.001$ vs CTRL) as well as activated leukocytes $(P<0.01$ vs CTRL).

Leukocyte profiling of MBC-treated animals was associated in cultured WAT explants with decreased levels of pro-inflammatory cytokines and chemokines, such as IL-6, TNF- $\alpha$ and IFN- $\gamma(P<0.001$ vs CTRL and LGG), IL-17A ( $P<0.001$ vs LGG), IP-10 ( $P<0.01$ vs LGG and $P<0.05$ vs CTRL), GM-CSF, and RANTES $(P<0.05$ vs CTRL). Reduced levels were also observed in WAT leukocytes of LGG-supplemented mice, but they related to a smaller subset of pro-inflammatory cytokines, namely IL-6 and IFN- $\gamma(P<0.001$ vs CTRL), IL-17A,

Table 2 Serum metabolic measurements in MBC, LGG, or CTRL mice

\begin{tabular}{llll}
\hline & MBC & LGG & CTRL \\
\hline Glucose $(\mathrm{mg} / \mathrm{dl})$ & $107.42 \pm 47.74$ & $72.97 \pm 7.64$ & $86.91 \pm 11.43$ \\
$\begin{array}{l}\text { Triglycerides } \\
(\mathrm{mg} / \mathrm{dl})\end{array}$ & $147.12^{*} \pm 65.91$ & $163.21^{\#} \pm 69.23$ & $316.98 \pm 142.39$ \\
$\begin{array}{l}\mathrm{HDL} \text { cholesterol } \\
(\mathrm{mg} / \mathrm{dl})\end{array}$ & $155.91^{* *} \pm 16.13$ & $137.29^{*} \pm 25.21$ & $108.86 \pm 12.91$ \\
$\begin{array}{l}\mathrm{LDL} \text { cholesterol } \\
(\mathrm{mg} / \mathrm{dl})\end{array}$ & $92.75^{\#} \pm 19.06$ & $105.77 \pm 48.03$ & $132.83 \pm 15.09$ \\
$\begin{array}{l}\text { Adiponectin } \\
(\mu \mathrm{g} / \mathrm{ml})\end{array}$ & $16.25 \pm 3.39$ & $17.46 \pm 4.87$ & $19.23 \pm 5.53$ \\
\hline${ }^{*} P<0.05$ versus $\mathrm{CTRL} ;{ }^{* *} P<0.001$ versus & $\mathrm{CTRL} ;{ }^{\#} P=0.05$ versus CTRL
\end{tabular}



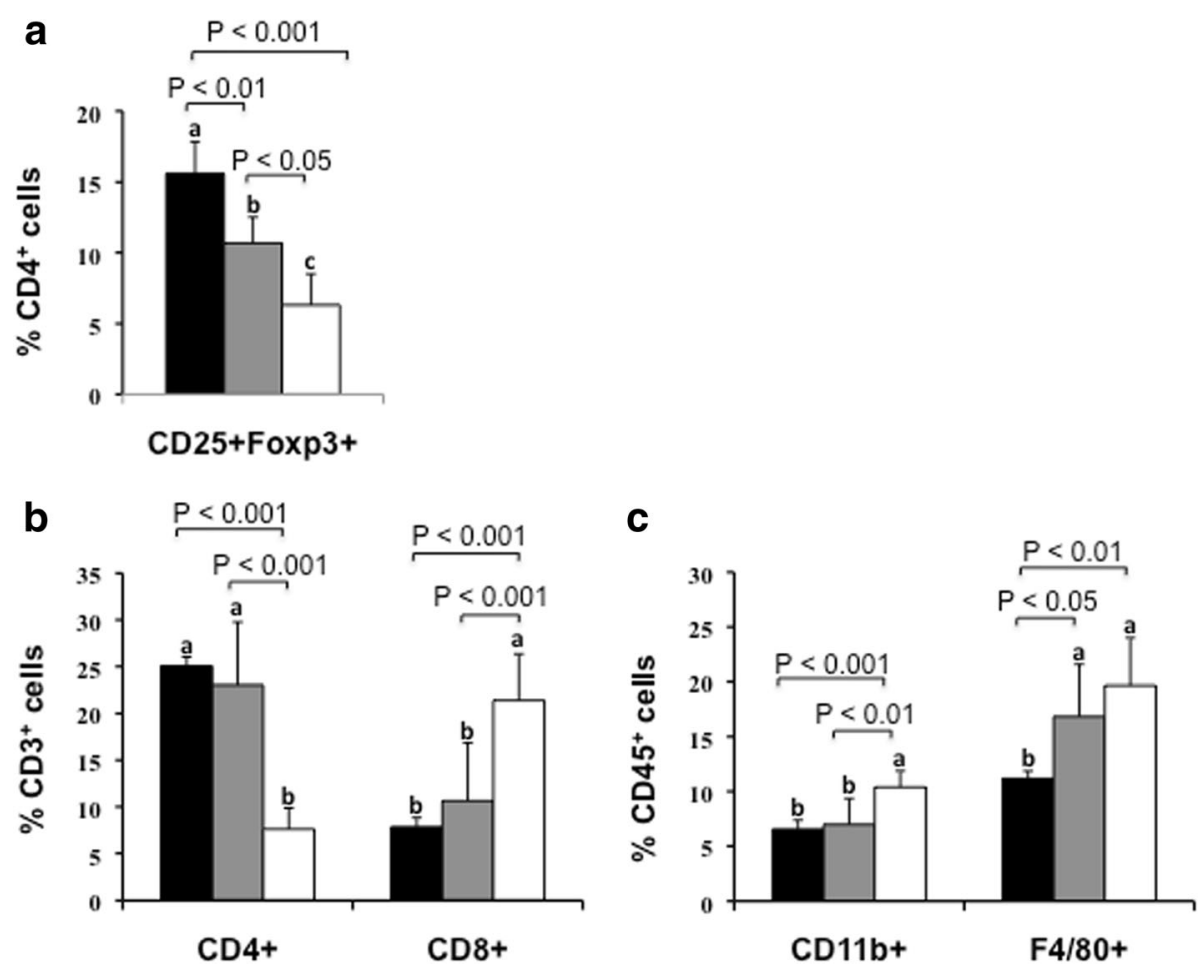

Fig. 2 Leukocyte subpopulations in epididymal WAT. The effect of bacterial supplementation on the frequency of WAT leukocyte subpopulations was analysed by flow cytometry. The percentage of CD25 Foxp3 $3^{+}$Treg cells was calculated on T lymphocyte gate (CD4+, a), CD $4^{+}$and $C D 8^{+}$cell subsets were calculated on lymphocyte gate $\left(\mathrm{CD}^{+}, \mathbf{b}\right)$, whereas $\mathrm{CD} 11 \mathrm{~b}^{+}$and $\mathrm{F} 4 / 80^{+}$cells were calculated on leukocyte gate $\left(\mathrm{CD} 45^{+}, \mathbf{c}\right) . \mathrm{Black}$ columns: MBC-supplemented mice; grey columns: LGG-supplemented; white columns: CTRL. Each column represents the mean \pm SD of nine mice. Means without a common letter significantly differ

and RANTES $(P<0.001$ and $P<0.01$ vs CTRL, respectively) (Fig. 3). No significant differences were observed among mice groups for the two anti-inflammatory cytokines IL-4 and IL-10 nor for pro-inflammatory IL-23 (data not shown).

Considering the dynamic and inherently multivariate nature of the immune response, WAT immunological profiles were further explored by principal component analysis (PCA) (Table 3). The first three principal components accounted for $64.15 \%$ of the overall variance, with individual values of 33.81, 19.47, and $10.87 \%$ for PC1, PC2, and PC3, respectively. The most informative score plot was the PC1/PC2 shown in Fig. 4, where PC1 was responsible for clearly discriminating $\mathrm{MBC}$ samples from LGG and CTRL samples. The variables mostly contributing to such discrimination are identified by higher loading values on PC1 (presented in italic characters in Table 3), indicating significant correlation between $\mathrm{PC} 1$ and the specific variable. In particular: PC1 shows strong significant inverse correlation with the pro-inflammatory markers $\mathrm{CD} \mathrm{CD}^{+}(r=-0.813)$, $\mathrm{CD}_{11 \mathrm{~b}}{ }^{+}(r=-0.727), \mathrm{F} 4 / 80^{+}(r=-0.804), \mathrm{IL}-6(r=-$ $0.669)$, TNF- $\alpha(r=-0.660)$, and GM-CSF $(r=-0.544)$ and significant direct correlation with the anti- inflammatory markers $\mathrm{CD} \mathrm{CD} 4^{+}(r=0.778)$ and $\mathrm{CD} 4 \mathrm{CD} 25^{+}(r=0.819)$. However, a tendency of the LGG and CTRL samples to separate into two distinct clusters is also observed (Fig. 4). PC2, on the other hand, discriminates a subgroup of CTRL mice showing both pro- and anti-inflammatory features. These features are highlighted by the most discriminative variables: the pro-inflammatory cytokines IP-10 $(r=0.842)$ and IFN- $\gamma$ $(r=0.587)$ and the anti-inflammatory markers IL-4 $(r=0.733)$ and IL-10 $(r=0.763)$ (Table 3).

\section{Impact of bacterial supplementation on gut microbiota profiles}

Next-generation sequencing (NGS) of 16S rDNA from faecal samples of treated or control mice was used to retrieve information on the bacterial relative abundance at time points $\mathrm{t} 0, \mathrm{t} 15$, and $\mathrm{t} 105$. Taxonomical assignment and read abundance estimates for all detected operational taxonomic units (OTUs) are reported in Fig. 5 at the phylum level, while the corresponding profiles at the species level are listed in Additional file 1: Table S1. As expected, Bacteroidetes and Firmicutes were detected as predominant bacterial phyla, with different relative proportions related to the time points analysed (Fig. 5). 


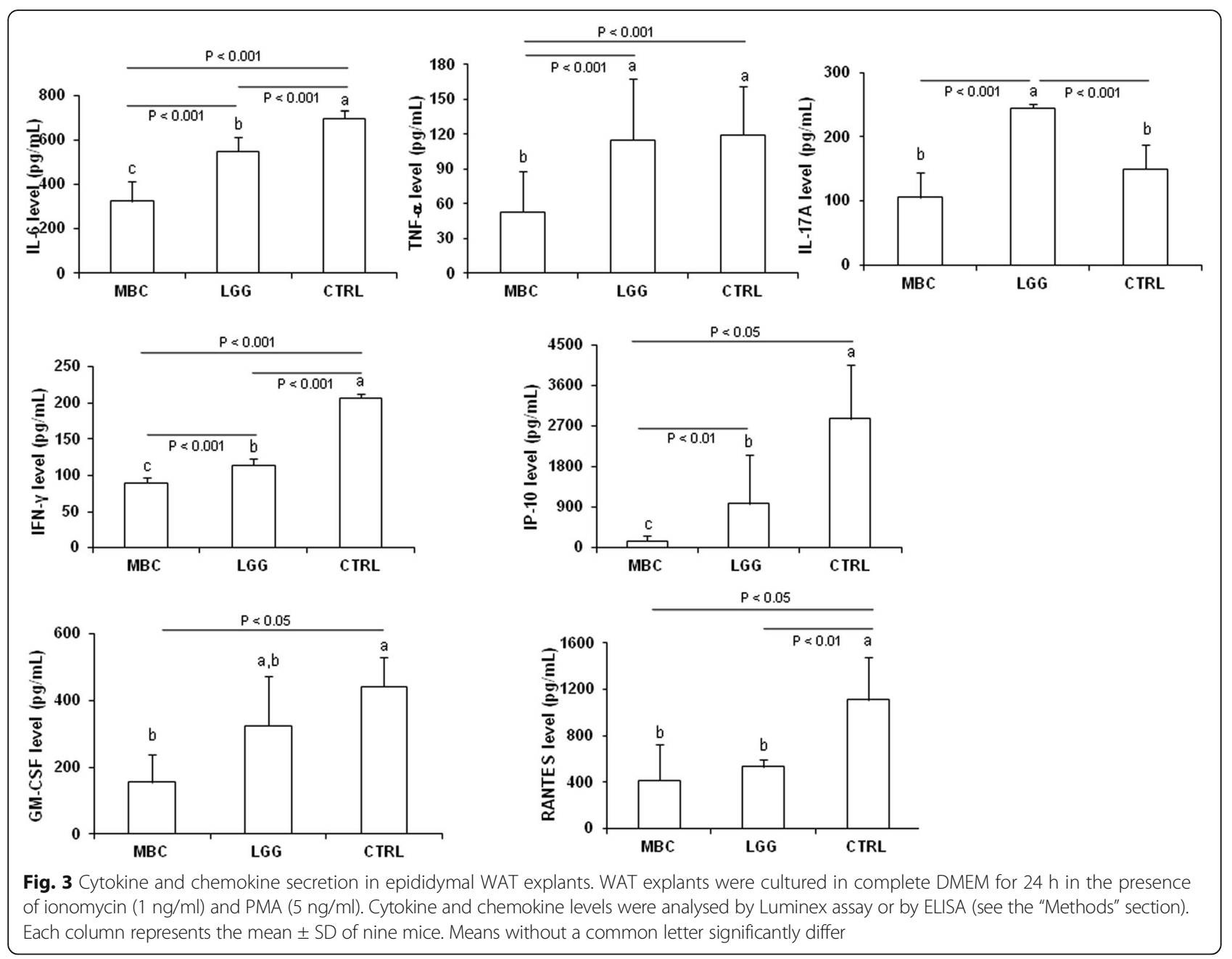

Notably, all three experimental groups displayed statistically significant increase in the Firmicutes/Bacteroidetes ratio at the final time point as compared to the beginning of the HFD treatment (t105 vs t15: $P<0.001$ for MBC and LGG; $P<0.05$ for CTRL). These altered ratios were also accompanied by decreased microbial biodiversity, measured by the Chaol and Shannon indices (data not shown). Differences in the overall composition of the faecal bacterial community were further analysed using the UniFrac phylogeny-based metric [40]. Principal Coordinates Analysis (PCoA) confirmed clustering of bacterial species according to sampling time. The first three principal components accounted for $41 \%$ of the overall variance, with individual values of 23,10 , and $8 \%$ for $\mathrm{PC} 1, \mathrm{PC} 2$, and $\mathrm{PC} 3$, respectively. The most informative score plot was the PC1/PC2, shown in Fig. 6. A clear difference was observed between the initial ( $\mathrm{t} 0$, t15) and final (t105) time points (Fig. 6a), while no difference could be observed among the three experimental conditions when samples were grouped according to supplementation type (Fig. 6b). However, it is worth noting that both $L$. delbrueckii and Leuc. lactis species, representing two major components of the $\mathrm{MBC}$ microbiota $[29,30]$, were detected exclusively in faecal samples of MBC-supplemented mice, although at very low abundance (Additional file 1: Table S1).

\section{Discussion}

In this work, we investigated the effects of a complex foodborne bacterial community (MBC microbiota) on obesity-associated inflammation and gut microbiota composition in a HFD-induced obese mouse model. The cultivable $\mathrm{LAB}$ component of MBC microbiota, selected by growth in MRS medium, was extracted from a fermented unripened cheese especially rich in live titres of LAB species [29] dominated by L. fermentum, L. delbrueckii, and Leuc. lactis [30] whose strains have often been associated with probiotic features [42]. The rationale for supplementing mice with the microbial consortium was based on the highly biodiverse nature of foodborne strains in fermented dairies, including several LAB strains of environmental origin with beneficial, 
Table 3 PCA loadings relative to the first two principal components from WAT immunological profiles of MBC, LGG, and CTRL mice

\begin{tabular}{lll}
\hline & $P C 1$ & $P C 2$ \\
\hline CD8 $^{+}$ & 0.3609 & -0.1459 \\
CD4 $^{+}$ & -0.3455 & 0.0893 \\
CD4CD25 $^{+}$ & -0.3635 & 0.0669 \\
CD11 ${ }^{+}$ & 0.3228 & -0.1137 \\
F4/80 & \\
GM-CSF & 0.3569 & -0.0599 \\
RANTES & 0.2416 & -0.1880 \\
IL-23 & 0.1786 & -0.1534 \\
IP-10 & 0.1371 & -0.1230 \\
IL-4 & 0.1248 & 0.4924 \\
IL-10 & -0.0169 & 0.4287 \\
IL-6 & 0.0720 & 0.4465 \\
IL-17A & 0.2973 & 0.3429 \\
IFN- $\gamma$ & 0.1223 & 0.0648 \\
TNF- $a$ & 0.2593 & 0.3435 \\
\hline
\end{tabular}

Positive or negative values indicate a direct or inverse correlation between variables and PCs, respectively. Loading values associated to significant Pearson's correlation coefficients (reported in the text) are indicated in italics

although yet uncharacterised, features [28]. Their combined metabolic functions and metabolites have been suggested to exert positive effects on host physiology through synergistic mechanisms, more efficiently than single strain supplementation [43]. However, the probiotic capacity of mixed foodborne microbial consortia has been gaining consideration only recently [44-46].

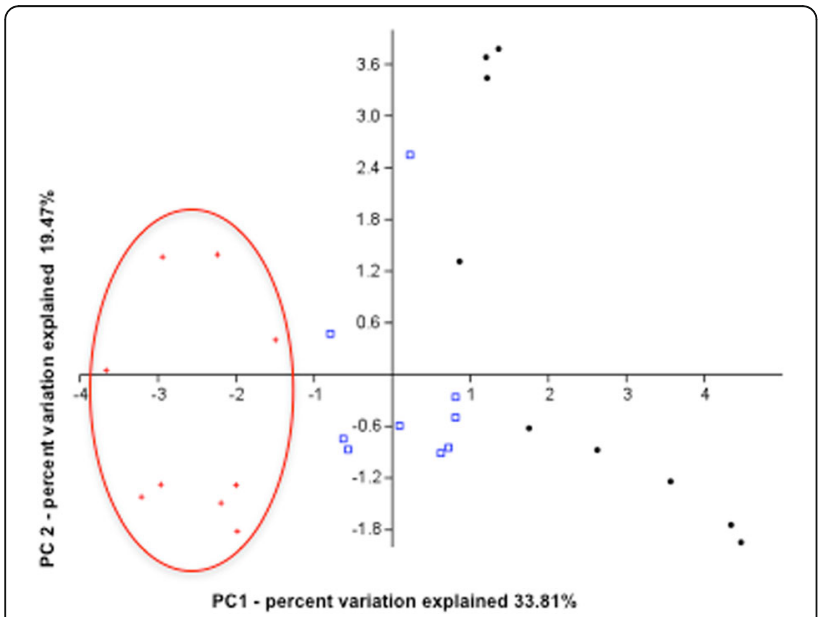

Fig. 4 PCA plot from epididymal WAT immunological profiles. PC1/PC2 score plot showing the distribution of samples in reduced PC1/PC2 space. The percentage variation explained by the plotted principal components is indicated. Symbols refer to individual mice. Red crosses: MBC-supplemented mice; blue squares: LGGsupplemented; black dots: CTRL
Moreover, most published work report supplementation with single bacterial strains, and only few studies compared multi-strain probiotic mixtures to investigate possible synergistic interactions [47]. We chose to run a parallel group of mice for comparison, supplemented with the single probiotic strain GG of Lactobacillus rhamnosus that was shown to exert positive effects on obesity-related inflammation in mice and humans [31].

The obese phenotype was induced in C57BL/6J mice by feeding a $45 \%$ HFD for 3 months, resulting in weight gain in all experimental groups irrespective of bacterial supplementation type. Many other studies report decreased body weight gain following probiotic supplementation [31, 48]. Although we detected constant weight gain in all mice groups, decreased epididymal WAT weight was evident following oral administration of MBC microbiota as compared to the other mice groups, as well as a more pronounced anti-inflammatory effect than LGG supplementation. Decreased inflammation and amelioration of obesity-related metabolic and immunological dysfunctions were previously observed with bacterial supplementation of HFD-fed mice $[49,50]$, but they were not accompanied by WAT weight reduction. WAT is considered the main contributor to development of the obesity-associated low-grade chronic systemic inflammatory state, which is characterised by an imbalanced cytokine network with increased production of several pro-inflammatory mediators. Epididymal WAT, like other intra-abdominal WAT depots, is now recognised to have a more negative impact on health than subcutaneous WAT [51], and its decreased weight following MBC supplementation further highlights a higher efficacy of this complex microbial community in supporting healthy metabolism. The specific antiinflammatory effects observed in our study involved decreased levels of the pro-inflammatory cytokines IL-6 and IFN- $\gamma$ and of the chemokines IP-10 and RANTES in cultured WAT explants of LGG-supplemented mice, while $\mathrm{MBC}$-treated animals displayed stronger decrease in the expression of a broader panel of pro-inflammatory cytokines and chemokines, namely IL-6, TNF- $\alpha$, IL-17A, IFN$\gamma$, IP-10, GM-CSF, and RANTES. Other studies using single probiotic strains or multi-strain mixtures observed decreased expression of some of these markers $[48,50,52]$. IL- 6 and TNF- $\alpha$ are the main cytokines produced by proinflammatory macrophages in obese adipose tissue, whereas RANTES and IP-10 are important lymphocyte and macrophage chemo-attractants [9]. IFN- $\gamma$ is secreted by infiltrating $\mathrm{CD}^{+} \mathrm{T}$ cells, thus contributing to the critical events driving adipose tissue inflammation [53]. Regarding IL-17, it was suggested that obesity predisposes to selective expansion of the Th17 subclass of T lymphocytes, producing high levels of IL-17 in an IL-6-dependent process [54]. The cytokine GM-CSF, although not 


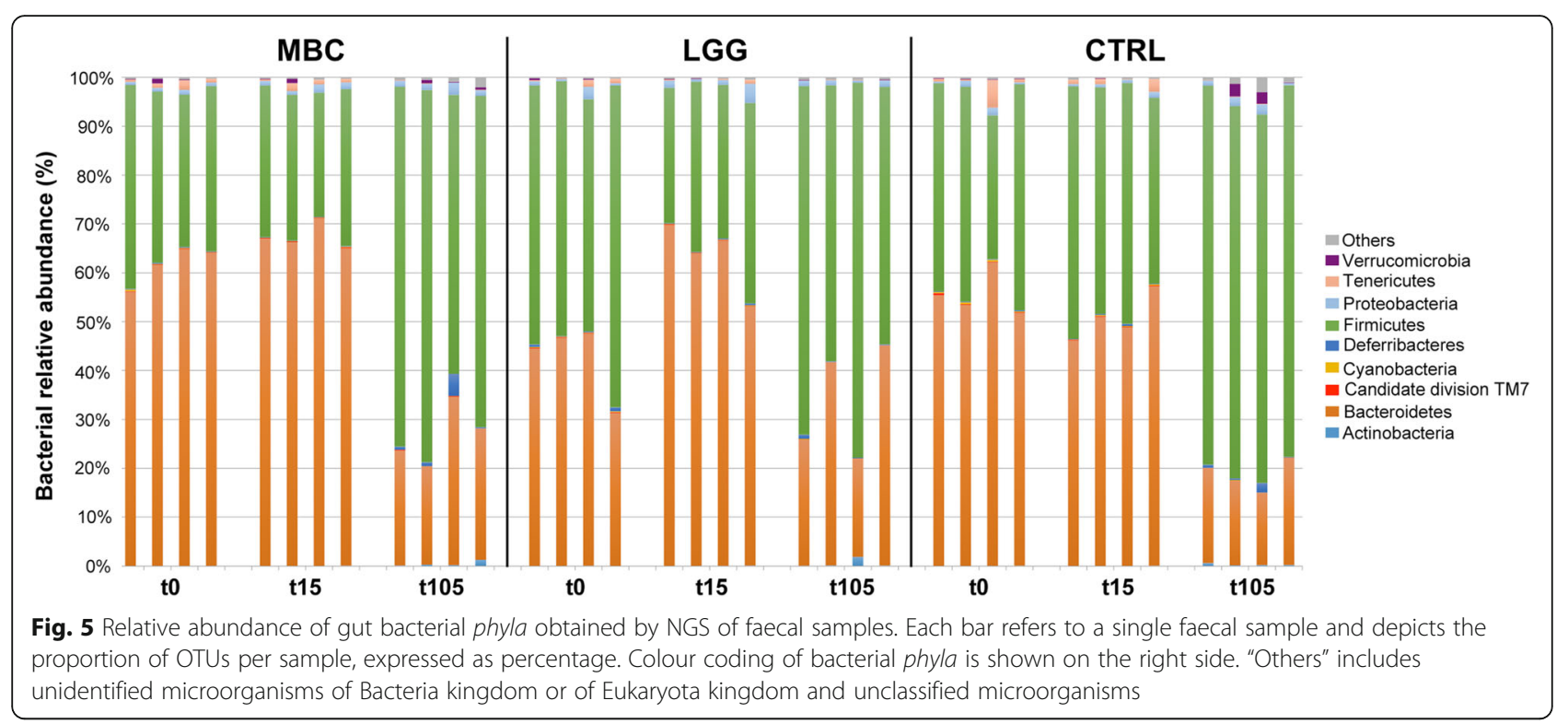

frequently measured in studies addressing probioticdependent immunomodulation in obesity, was reported to increase in the serum of obese mice [55].

The positive effects exerted by $\mathrm{MBC}$ supplementation on the overall profile of WAT inflammatory cytokines and chemokines were also associated to improved balance between the major sub-populations of immune cells, as revealed by reduced percentage of proinflammatory $\mathrm{CD}^{+} \mathrm{T}$ lymphocytes, activated leukocytes and macrophages, and increased $\mathrm{CD} 4^{+} \mathrm{T}$ lymphocytes

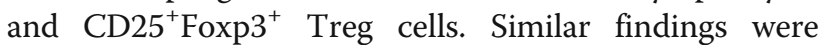
reported in other tissues following Bifidobacterium pseudocatenulatum supplementation [50], in the adipose tissue after Lactobacillus gasseri supplementation [56], or using a probiotic mixture of L. rhamnosus and Bifidobacterium animalis subsp. lactis [48]. Treg cells are highly represented in the WAT of lean mice, and they are essential for the maintenance of an anti-inflammatory environment in the absence of obesity. Treg cell number has been shown to decrease in the WAT of obese mice, contributing to worsen the inflammatory state $[10,11]$. The increased Treg cell number that we observe after MBC supplementation is a result of particular relevance, considering that selective modulation of this population was shown to be tightly related to the level of obesityassociated inflammation [10].
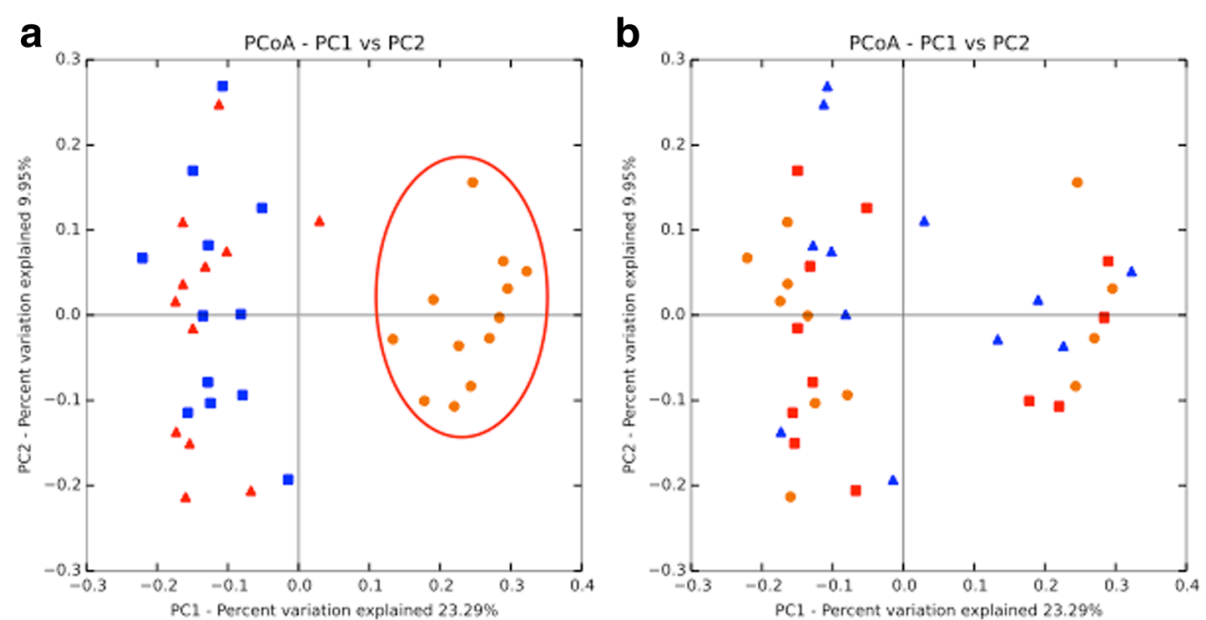

Fig. 6 PCoA plots of unweighted UniFrac distance matrix. PC1/PC2 score plot showing the distribution of samples. The same plots are shown in each panel, with symbols referring to individual samples, but colour coding of each sample refers to time points in a ( $\mathrm{t} 0=$ red triangles, $\mathrm{t} 15=$ blue squares, $\mathrm{t} 105=$ orange circles) or treatment type in $\mathbf{b}(\mathrm{CTRL}=$ red triangles, $\mathrm{LGG}=$ blue squares, $\mathrm{MBC}=$ orange circles). The percentage variation explained by the plotted principal coordinates is indicated in the axis legend. Score values shown along the axes represent the proportion of dissimilarities captured by each axis 
The anti-inflammatory effects occurring with MBC supplementation were even more evident following PCA analysis of the datasets, which clearly discriminated $\mathrm{MBC}$ samples from LGG and CTRL samples along the first principal component axis. This confirms the key role of the immune cell subpopulations, as well as of the cytokines GM-CSF, IL-6, and TNF- $\alpha$, as the most important variables contributing to the discrimination. Separation of the LGG and CTRL samples into two distinct clusters was highlighted only as a trend. These effects were accompanied by positive changes in the expression of lipid metabolism biomarkers in the MBC-supplemented group, with decreased circulating levels of triglycerides, increased HDL-cholesterol levels, and a trend toward decreasing LDL cholesterol. Higher levels of circulating HDL cholesterol were also observed in the LGG mice group, in line with previous reports on supplementation with single probiotics or mixtures $[31,48,50]$.

Interaction with the host metagenome is considered an important aspect in probiotic-mediated immune stimulation [22, 57]. We analysed faecal microbiota biodiversity in treated mice by NGS of $16 \mathrm{~S}$ rDNA. Our results confirmed that gut microbiota composition was indeed affected by HFD, leading to the establishment of an increased Firmicutes/Bacteroidetes ratio typical of the obesity pattern [58]. Bacterial supplementation was not able to overcome HFD-induced effects on gut microbial profile, as no substantial modifications in faecal microbiota composition could be observed over time by NGS. The overriding effect of HFD on microbial biodiversity was also confirmed by advanced multivariate statistical analysis, namely Principal Coordinates Analysis (PCoA), revealing no specific clustering of bacterial species according to supplementation type, while highlighting a clear variation of microbial composition at the end of the experimental period in all mice groups. Other studies reported different extent of alterations in resident gut microbiota profile following probiotic treatment of HFD-fed mice [48, 50, $59,60]$, but the studies are not always comparable due to different experimental designs (duration of treatment, percent dietary fat, etc.) and experimental approaches employed for microbial profiling (i.e. NGS, qPCR). In our study, the high sensitivity of NGS allowed to detect two of the three predominant species characterising the MBC-derived microbiota, namely $L$. delbrueckii and Leuc. lactis, although with low relative abundance in the faecal microbiome of supplemented mice. These two species may thus be able to colonise the gut of supplemented mice more efficiently. Gut colonisation capacity of some components of MBCderived microbiota was also shown in the simple model organism Caenorhabditis elegans [30]. On the other hand, the L. rhamnosus species that includes the
LGG strain was undetectable in faecal microbiomes of LGG-treated mice. Conflicting results concerning LGG colonisation capacity have been reported in the literature. Park et al. recently observed decreased Lactobacillus relative abundances in the murine gut, including the LGG strain, following LAB supplementation [59], while in another report of orally administered LGG to knockout (ApoE-/-) mice fed HFD, $L$. rhamnosus could be recovered by faecal dilution and plating [61]. Nevertheless, several reports indicate that oral administration of specific bacteria can exert beneficial effects on the host even in the absence of colonisation [59, 62-64].

Taken together, our results suggest that supplementation with a biodiverse foodborne bacterial consortium can exert beneficial effects on obesity-associated inflammation and health-related parameters more effectively than single probiotic strain supplementation. A recent report by Sonnenburg et al. clearly shows that dietary perturbations can lead to permanent loss of specific gut bacterial taxa, due to negative selection of metabolic activities that become unnecessary under imbalanced dietary regimens [65]. These results point at limitations in microbiota resilience occurring under extreme conditions, such as HFD-induced obesity, where the alterations cannot be reversed by simple dietary intervention if not accompanied by specific bacterial supplementation aimed at restoring the lost taxa. Foodborne bacteria could play a key role in this respect, and to the best of our knowledge, this is among the very few reports evaluating the impact of a complex microbial consortium naturally occurring in a traditional fermented food on host physiology.

\section{Conclusions}

Our results demonstrate a stronger effect of a mixed microbial consortium vs single-strain probiotic supplementation in ameliorating HFD-induced inflammation in the WAT of obese mice. The present study highlights the importance of considering complex foodborne microbial consortia naturally occurring in fermented products for human consumption as potential probiotic vectors. It also points at the importance of coupling multivariate to univariate statistical analysis for better understanding of the key factors responsible for probiotic effects. The observed immunomodulatory activity exerted by the MBC-derived microbiota suggests synergistic interactions of microbial strains of environmental origin, present within the foodborne consortium. More studies are needed to further investigate the role of dietary microbes with yet uncharacterised probiotic effect, aimed also at identifying novel, under-represented strains which could be unique to the foodborne microbiota. 


\section{Additional file}

Additional file 1: Table S1. Complete results of the identification, at the species level or, where not possible, at higher taxonomic ranks, of the sequences obtained by next-generation sequencing of faecal mice samples, expressed as percentage. Species representing the components of MBC microbiota are highlighted in bold. (XLSX 96 kb)

\section{Abbreviations}

CFU: Colony-forming units; CTRL: Control; GM-CSF: Granulocyte macrophagecolony stimulating factor; HFD: High fat diet; IFN: Interferon; IL: Interleukin; IP: Interferon gamma-induced protein; LAB: Lactic acid bacteria; LGG: L. rhamnosus GG; MBC: Mozzarella di Bufala Campana; MRS: De Man Rogosa Sharpe medium; NGS: Next-generation sequencing; OTUs: Operational taxonomic units; PCA: Principal component analysis; PCoA: Principal Coordinates Analysis; RANTES: Regulated on Activation-Normal T cell Expressed and Secreted; TNF: Tumour necrosis factor; Treg: Regulatory T cells; WAT: White adipose tissue

\section{Acknowledgements}

The Authors wish to thank Kariklia Pascucci for her kind support in daily lab work, Dr. Andrea Ciolfi for his valuable assistance in managing the NGS data, and Dr. Fausta Natella and Dr. Gianni Pastore for their helpful suggestions on the statistical analysis.

\section{Funding}

This work was funded in part by the Italian Ministry of Agriculture, Food \& Forestry Policies (MiPAAF), with grant "MEDITO" (DM 12487/7303/11) and with national support to the JPI-HDHL "ENPADASI" project.

\section{Availability of data and materials}

Raw NGS data are available at the EMBL-EBI European Nucleotide Archive (ENA) [66], under the study accession number PRJEB20801.

\section{Authors' contributions}

$M R, G P$, and $C D$ conceived and designed the experiments. MR, PZ, BG, $A F$, and $C D$ performed the experiments. $M R$ and $C D$ analysed the data and supervised all data analyses. GP contributed reagents/materials/ analysis tools. MR, GP, and CD wrote the paper. RR did the animal experiments/treatments. MR and AF performed the immunological analysis. $P Z, B G$, and $C D$ performed the microbiological analysis. $C D$ analysed the microbiota sequencing data. All authors read and approved the final manuscript.

\section{Ethics approval}

All experimental procedures involving animals complied with the European Guidelines for the Care and Use of Animals for Research Purposes (Directive 2010/63/EU), and protocols were approved by the Ethical Committee of the Food and Nutrition Research Center and by the National Health Ministry, General Direction of Animal Health and Veterinary Drugs (agreement no 201/2015-PR).

\section{Consent for publication}

Not applicable.

\section{Competing interests}

The authors declare that they have no competing interests.

\section{Publisher's Note}

Springer Nature remains neutral with regard to jurisdictional claims in published maps and institutional affiliations.
Received: 24 May 2017 Accepted: 13 August 2017

Published online: 04 October 2017

\section{References}

1. World Health Organization. Obesity and overweight fact sheet N 311updated March 2011 2011, http://www.who.int/mediacentre/factsheets/ fs311/en/index.html.

2. Ng M, Fleming T, Robinson M, Thomson B, Graetz N, Margono C, Mullany EC, Biryukov S, Abbafati C, Abera SF, et al. Global, regional, and national prevalence of overweight and obesity in children and adults during 1980-2013: a systematic analysis for the Global Burden of Disease Study 2013. Lancet. 2014;384:766-81.

3. Serra-Majem L, Bautista-Castano I. Etiology of obesity: two "key issues" and other emerging factors. Nutr Hosp. 2013;28(Suppl 5):32-43.

4. Crino M, Sacks G, Vandevijvere S, Swinburn B, Neal B. The influence on population weight gain and obesity of the macronutrient composition and energy density of the food supply. Curr Obes Rep. 2015:4:1-10.

5. Shabana HS. Obesity, more than a 'cosmetic' problem. Current knowledge and future prospects of human obesity genetics. Biochem Genet. 2016;54:1-28.

6. Cao H. Adipocytokines in obesity and metabolic disease. J Endocrinol. 2014;220:T47-59.

7. Khan Mt Joseph F. Adipose tissue and adipokines: the association with and application of adipokines in obesity. Scientifica (Cairo). 2014;2014:328592.

8. Maury E, Brichard SM. Adipokine dysregulation, adipose tissue inflammation and metabolic syndrome. Mol Cell Endocrinol. 2010;314:1-16.

9. Huh JY, Park YJ, Ham M, Kim JB. Crosstalk between adipocytes and immune cells in adipose tissue inflammation and metabolic dysregulation in obesity. Mol Cells. 2014;37:365-71.

10. Feuerer M, Herrero L, Cipolletta D, Naaz A, Wong J, Nayer A, Lee J, Goldfine $A B$, Benoist C, Shoelson S, Mathis D. Lean, but not obese, fat is enriched for a unique population of regulatory $T$ cells that affect metabolic parameters. Nat Med. 2009;15:930-9.

11. Kucharska AM, Pyrzak B, Demkow U. Regulatory T cells in obesity. Adv Exp Med Biol. 2015;866:35-40.

12. Gyllenhammer LE, Lam J, Alderete TL, Allayee H, Akbari O, Katkhouda N, Goran MI. Lower omental t-regulatory cell count is associated with higher fasting glucose and lower beta-cell function in adults with obesity. Obesity (Silver Spring). 2016;24:1274-82.

13. Alberti KG, Zimmet P, Shaw J. The metabolic syndrome-a new worldwide definition. Lancet. 2005;366:1059-62.

14. Schaffler A, Muller-Ladner U, Scholmerich J, Buchler C. Role of adipose tissue as an inflammatory organ in human diseases. Endocr Rev. 2006;27:449-67.

15. Sam S, Haffner S, Davidson MH, D'Agostino RB Sr, Feinstein S, Kondos G, Perez A, Mazzone T. Relation of abdominal fat depots to systemic markers of inflammation in type 2 diabetes. Diabetes Care. 2009;32:932-7.

16. Rocha VZ, Folco EJ. Inflammatory concepts of obesity. Int J Inflam. 2011; 2011:529061.

17. Bell DS. Changes seen in gut bacteria content and distribution with obesity: causation or association? Postgrad Med. 2015;127:863-8.

18. Cani PD, Osto M, Geurts L, Everard A. Involvement of gut microbiota in the development of low-grade inflammation and type 2 diabetes associated with obesity. Gut Microbes. 2012;3:279-88.

19. Ley RE, Backhed F, Turnbaugh P, Lozupone CA, Knight RD, Gordon II. Obesity alters gut microbial ecology. Proc Natl Acad Sci U S A. 2005;102:11070-5

20. John GK, Mullin GE. The gut microbiome and obesity. Curr Oncol Rep. 2016;18:45.

21. Dahiya DK, Renuka PM, Shandilya UK, Dhewa T, Kumar N, Kumar S, Puniya AK, Shukla P. Gut microbiota modulation and its relationship with obesity using prebiotic fibers and probiotics: a review. Front Microbiol. 2017:8:563.

22. Sanchez B, Delgado S, Blanco-Miguez A, Lourenco A, Gueimonde M, Margolles A. Probiotics, gut microbiota, and their influence on host health and disease. Mol Nutr Food Res. 2017;61

23. Gauffin Cano P, Santacruz A, Moya A, Sanz Y. Bacteroides uniformis CECT 7771 ameliorates metabolic and immunological dysfunction in mice with high-fat-diet induced obesity. PLoS One. 2012;7:e41079.

24. Ji YS, Kim HN, Park HJ, Lee JE, Yeo SY, Yang JS, Park SY, Yoon HS, Cho GS, Franz CM, et al. Modulation of the murine microbiome with a concomitant anti-obesity effect by Lactobacillus rhamnosus GG and Lactobacillus sakei NR28. Benef Microbes. 2012;3:13-22. 
25. Takemura N, Okubo T, Sonoyama K. Lactobacillus plantarum strain no. 14 reduces adipocyte size in mice fed high-fat diet. Exp Biol Med (Maywood). 2010;235:849-56.

26. Shiby VK, Mishra HN. Fermented milks and milk products as functional foods-a review. Crit Rev Food Sci Nutr. 2013;53:482-96.

27. Wolfe BE, Dutton RJ. Fermented foods as experimentally tractable microbial ecosystems. Cell. 2015;161:49-55.

28. Tamang JP, Watanabe K, Holzapfel WH. Review: diversity of microorganisms in global fermented foods and beverages. Front Microbiol. 2016;7:377.

29. Devirgiliis C, Caravelli A, Coppola D, Barile S, Perozzi G. Antibiotic resistance and microbial composition along the manufacturing process of Mozzarella di Bufala Campana. Int J Food Microbiol. 2008;128:378-84.

30. Zanni E, Laudenzi C, Schifano E, Palleschi C, Perozzi G, Uccelletti D, Devirgiliis C. Impact of a complex food microbiota on energy metabolism in the model organism Caenorhabditis elegans. Biomed Res Int. 2015;2015:621709.

31. Kobyliak N, Conte C, Cammarota G, Haley AP, Styriak I, Gaspar L, Fusek J, Rodrigo L, Kruzliak P. Probiotics in prevention and treatment of obesity: a critical view. Nutr Metab (Lond). 2016;13:14.

32. Kim B, Park KY, Ji Y, Park S, Holzapfel W, Hyun CK. Protective effects of Lactobacillus rhamnosus GG against dyslipidemia in high-fat diet-induced obese mice. Biochem Biophys Res Commun. 2016;473:530-6.

33. Orr JS, Kennedy AJ, Hasty AH. Isolation of adipose tissue immune cells. J Vis Exp. 2013:e50707.

34. Strissel KJ, DeFuria J, Shaul ME, Bennett G, Greenberg AS, Obin MS. T-cell recruitment and Th1 polarization in adipose tissue during diet-induced obesity in C57BL/6 mice. Obesity (Silver Spring). 2010;18:1918-25.

35. Wagner Mackenzie B, Waite DW, Taylor MW. Evaluating variation in human gut microbiota profiles due to DNA extraction method and inter-subject differences. Front Microbiol. 2015;6:130

36. Milani C, Hevia A, Foroni E, Duranti S, Turroni F, Lugli GA, Sanchez B, Martin $R$, Gueimonde M, van Sinderen D, et al. Assessing the fecal microbiota: an optimized ion torrent 165 rRNA gene-based analysis protocol. PLoS One. 2013;8:e68739.

37. Caporaso JG, Kuczynski J, Stombaugh J, Bittinger K, Bushman FD, Costello EK, Fierer N, Pena AG, Goodrich JK, Gordon Jl, et al. QIIME allows analysis of high-throughput community sequencing data. Nat Methods. 2010;7:335-6.

38. Edgar RC. Search and clustering orders of magnitude faster than BLAST. Bioinformatics. 2010;26:2460-1.

39. Quast C, Pruesse E, Yilmaz P, Gerken J, Schweer T, Yarza P, Peplies J, Glockner FO. The SILVA ribosomal RNA gene database project: improved data processing and web-based tools. Nucleic Acids Res. 2013;41:D590-6.

40. Lozupone C, Knight R. UniFrac: a new phylogenetic method for comparing microbial communities. Appl Environ Microbiol. 2005:71:8228-35.

41. Hammer $\varnothing$, Harper DAT, Ryan PD. PAST: paleontological statistics software package for education and data analysis. Palaeontol Electron. 2001;4:9pp.

42. Tamang JP, Shin DH, Jung SJ, Chae SW. Functional properties of microorganisms in fermented foods. Front Microbiol. 2016;7:578.

43. Bordoni A, Danesi F, Dardevet D, Dupont D, Fernandez AS, Gille D, Nunes Dos Santos C, Pinto P, Re R, Remond D, et al. Dairy products and inflammation: a review of the clinical evidence. Crit Rev Food Sci Nutr. 2017:57:2497-525

44. Rosa DD, Dias MM, Grzeskowiak LM, Reis SA, Conceicao LL, Peluzio MD. Milk kefir: nutritional, microbiological and health benefits. Nutr Res Rev. 2017:1-15

45. Kim DH, Kim H, Jeong D, Kang IB, Chon JW, Kim HS, Song KY, Seo KH. Kefir alleviates obesity and hepatic steatosis in high-fat diet-fed mice by modulation of gut microbiota and mycobiota: targeted and untargeted community analysis with correlation of biomarkers. J Nutr Biochem. 2017:44:35-43.

46. Choi JW, Kang HW, Lim WC, Kim MK, Lee IY, Cho HY. Kefir prevented excess fat accumulation in diet-induced obese mice. Biosci Biotechnol Biochem. 2017;81:958-65

47. Foligne B, Parayre S, Cheddani R, Famelart MH, Madec MN, Ple C, Breton J, Dewulf J, Jan G, Deutsch SM. Immunomodulation properties of multispecies fermented milks. Food Microbiol. 2016;53:60-9.

48. Alard J, Lehrter V, Rhimi M, Mangin I, Peucelle V, Abraham AL, Mariadassou M, Maguin E, Waligora-Dupriet AJ, Pot B, et al. Beneficial metabolic effects of selected probiotics on diet-induced obesity and insulin resistance in mice are associated with improvement of dysbiotic gut microbiota. Environ Microbiol. 2016:18:1484-97.
49. Cano PG, Santacruz A, Trejo FM, Sanz Y. Bifidobacterium CECT 7765 improves metabolic and immunological alterations associated with obesity in high-fat diet-fed mice. Obesity (Silver Spring). 2013;21:2310-21.

50. Moya-Perez A, Neef A, Sanz Y. Bifidobacterium pseudocatenulatum CECT 7765 reduces obesity-associated inflammation by restoring the lymphocytemacrophage balance and gut microbiota sstructure in high-fat diet-fed mice. PLoS One. 2015;10:e126976.

51. Wajchenberg BL, Giannella-Neto D, da Silva ME, Santos RF. Depot-specific hormonal characteristics of subcutaneous and visceral adipose tissue and their relation to the metabolic syndrome. Horm Metab Res. 2002;34:616-21.

52. Novotny Nunez I, Maldonado Galdeano C, de Moreno de LeBlanc A, Perdigon G. Lactobacillus casei CRL 431 administration decreases inflammatory cytokines in a diet-induced obese mouse model. Nutrition. 2015;31:1000-7.

53. Nishimura S, Manabe I, Nagasaki M, Eto K, Yamashita H, Ohsugi M, Otsu M, Hara K, Ueki K, Sugiura S, et al. CD8+ effector T cells contribute to macrophage recruitment and adipose tissue inflammation in obesity. Nat Med. 2009;15:914-20.

54. Ahmed M, Gaffen SL. IL-17 in obesity and adipogenesis. Cytokine Growth Factor Rev. 2010;21:449-53.

55. Boi SK, Buchta CM, Pearson NA, Francis MB, Meyerholz DK, Grobe JL, Norian LA. Obesity alters immune and metabolic profiles. New insight from obeseresistant mice on high-fat diet. Obesity (Silver Spring). 2016;24:2140-9.

56. Ukibe K, Miyoshi M, Kadooka Y. Administration of Lactobacillus gasseri SBT2055 suppresses macrophage infiltration into adipose tissue in dietinduced obese mice. Br J Nutr. 2015;114:1180-7.

57. Le Barz M, Anhe FF, Varin TV, Desjardins Y, Levy E, Roy D, Urdaci MC, Marette A. Probiotics as complementary treatment for metabolic disorders. Diabetes Metab J. 2015:39:291-303.

58. Turnbaugh PJ, Backhed F, Fulton L, Gordon Jl. Diet-induced obesity is linked to marked but reversible alterations in the mouse distal gut microbiome. Cell Host Microbe. 2008;3:213-23.

59. Park $\mathrm{S}$, Ji $Y$, Jung HY, Park H, Kang J, Choi SH, Shin H, Hyun CK, Kim KT, Holzapfel WH. Lactobacillus plantarum HAC01 regulates gut microbiota and adipose tissue accumulation in a diet-induced obesity murine model. Appl Microbiol Biotechnol. 2017;101:1605-14

60. Gauffin-Cano PG, Santacruz A, Trejo FM, Sanz Y. Bifidobacterium CECT 7765 improves metabolic and immunological alterations associated with obesity in high-fat diet-fed mice. Obesity (Silver Spring). 2013;21:2310-21.

61. Chan YK, Brar MS, Kirjavainen PV, Chen Y, Peng J, Li D, Leung FC, El-Nezami $H$. High fat diet induced atherosclerosis is accompanied with low colonic bacterial diversity and altered abundances that correlates with plaque size, plasma A-FABP and cholesterol: a pilot study of high fat diet and its intervention with Lactobacillus rhamnosus GG (LGG) or telmisartan in ApoE-/- mice. BMC Microbiol. 2016;16:264

62. Thomas CM, Versalovic J. Probiotics-host communication: modulation of signaling pathways in the intestine. Gut Microbes. 2010;1:148-63.

63. Hsiao EY, McBride SW, Hsien S, Sharon G, Hyde ER, McCue T, Codelli JA Chow J, Reisman SE, Petrosino JF, et al. Microbiota modulate behavioral and physiological abnormalities associated with neurodevelopmental disorders. Cell. 2013;155:1451-63.

64. Bjerg AT, Sorensen MB, Krych L, Hansen LH, Astrup A, Kristensen M, Nielsen DS. The effect of Lactobacillus paracasei subsp. paracasei and L. casei W8(R) on blood levels of triacylglycerol is independent of colonisation. Benef Microbes. 2015:6:263-9.

65. Sonnenburg ED, Smits SA, Tikhonov M, Higginbottom SK, Wingreen NS, Sonnenburg JL. Diet-induced extinctions in the gut microbiota compound over generations. Nature. 2016;529:212-5

66. Leinonen R, Akhtar R, Birney E, Bower L, Cerdeno-Tarraga A, Cheng Y, Cleland I, Faruque N, Goodgame N, Gibson R, et al. The European Nucleotide Archive. Nucleic Acids Res. 2011;39:D28-31. 